\title{
Quantum decoupling transition in a one-dimensional Feshbach-resonant superfluid
}

\author{
Daniel E. Sheehy and Leo Radzihovsky \\ Department of Physics, University of Colorado, Boulder, CO, 80309
}

(Dated: May 26, 2005)

\begin{abstract}
We study a one-dimensional gas of fermionic atoms interacting via an s-wave molecular Feshbach resonance. At low energies the system is characterized by two Josephson-coupled Luttinger liquids, corresponding to paired atomic and molecular superfluids. We show that, in contrast to higher dimensions, the system exhibits a quantum phase transition from a phase in which the two superfluids are locked together to one in which, at low energies, quantum fluctuations suppress the Feshbach resonance (Josephson) coupling, effectively decoupling the molecular and atomic superfluids. Experimental signatures of this quantum transition include the appearance of an out-of-phase gapless mode (in addition to the standard gapless in-phase mode) in the spectrum of the decoupled superfluid phase and a discontinuous change in the molecular momentum distribution function.
\end{abstract}

Recent experimental advances [1] have led to a realization of paired superfluidity in degenerate atomic gases. It is driven by the atomic Feshbach resonance (FR) through a molecular state whose rest energy (detuning) $\nu$ can be adjusted with a magnetic field. The associated high tunability of interactions allows one to explore superfluidity in these systems ranging from the BCS regime of strongly overlapping Cooper pairs (for large positive detuning) to the BEC regime of dilute Bose-condensed molecules (for negative detuning) 2].

In all BEC-BCS crossover studies to date, it has been tacitly (correctly [3, 4]) assumed that the superfluid phases of the closed-channel Bose-condensed molecules and open channel Cooper-pairs are locked together by the FR coupling, with the superfluid at low-energies characterized by a single gapless Bogoliubov (0th sound) mode. In this Letter we show that in striking contrast, a one-dimensional (1d) resonantly interacting atomic gas [5, 6, 7], realized by a sufficiently high aspect ratio trap [8, 9], can exhibit a more interesting possibility. Namely, for a range of parameters quantum fluctuations, enhanced by the low dimensionality, suppress the FR coupling thereby leading to a quantum phase transition into a superfluid state where the Cooper-pair and molecular superfluids are decoupled. Our main findings are summarized by the phase diagram in Fig. 11 One striking experimental signature of this decoupling transition is the appearance of an out-of-phase gapless mode (in addition to the abovementioned standard gapless inphase mode) in the spectrum of the decoupled superfluid phase, that should be observable through Bragg spectroscopy [10]. Another signature is a jump across the transition in the exponent $\alpha$ characterizing the molecular momentum distribution function $n_{b}(k) \propto k^{-\alpha}$ with $\alpha_{\text {decoupled }}<\alpha_{\text {coupled }}$, measurable via time-of-flight images [8].

The decoupling transition can be seen through the bosonization representation of the molecular and atomic quantum fluids as acoustic charge and spin collective modes, with the FR interaction reducing to a Josephson coupling between phases of the molecular and Cooper- pair superfluids. Now, a sufficiently strong atomic repulsion (attainable through optical lattice engineering $[8$, 9, 11]), that "localizes" atom number can be tuned to enhance the canonically-conjugate superfluid phase fluctuations, to the point that the FR (Josephson) coupling is averaged away at low energies.

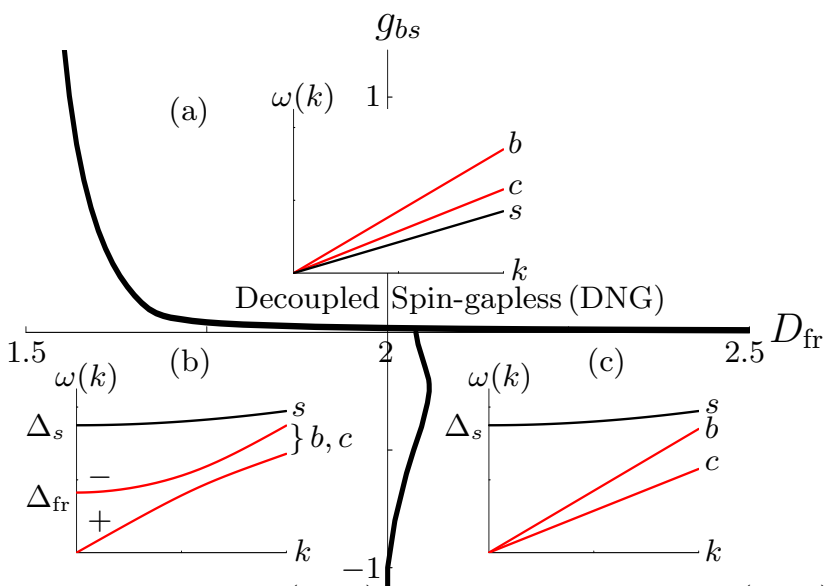

Coupled Spin-gapped (CSG) Decoupled Spin-gapped (DSG)

FIG. 1: (Color online) Main: Phase diagram as a function of the scaling dimension $D_{\mathrm{fr}}$ of the FR coupling and the direct atomic interaction strength $g_{b s}$ for $g_{\text {fr }}=0.19$. For attractive interactions, $g_{b s}<0$, fermion spins are gapped and the transition is between coupled and decoupled spin-gapped paired superfluids. For repulsive atomic interactions, $g_{b s}>0$, the transition is between the coupled spin-gapped state and a decoupled spin-gapless state. Insets a,b,c: Plots of spectrum for fermion spin $(s)$, fermion charge $(c)$, and boson charge $(b)$ excitations within these three phases.

This transition has strong connections to other interesting examples of fluctuation-driven decoupling of Josephson-coupled XY-models, that fall into the roughenning universality class 12. Most notably, these include the sliding phases of DNA-lipid complexes 13. and their quantum higher-dimensional Luttinger liquid (LL) generalizations 14], the latter a realization of a longsought-after LL power-law phenomenology in $d>1$.

We now demonstrate and explore the superfluid decoupling transition through a sketch of our detailed 
calculations 15. The appropriate model of a two-species $\sigma=\uparrow, \downarrow$ (two distinct values of an internal degree of freedom, e.g., hyperfine states) fermionic atomic gas interacting through a molecular FR is described by a Hamiltonian [2] $H=\sum_{k, \sigma} \epsilon_{k} c_{k \sigma}^{\dagger} c_{k \sigma}+\sum_{q}\left(\epsilon_{q} / 2+\nu\right) b_{q}^{\dagger} b_{q}+$ $H_{\text {fr }}+H_{\text {int }}$, where $\epsilon_{k}=\hbar^{2} k^{2} / 2 m, \nu$ is the FR detuning, and $H_{\text {int }}$ is the direct fermionic and bosonic interactions.

The FR interaction

$$
H_{\mathrm{fr}}=-\tilde{g}_{\mathrm{fr}} \int d^{d} x\left(c_{\uparrow}^{\dagger}(x) c_{\downarrow}^{\dagger}(x) b(x)+h . c .\right),
$$

describes the interconversion between a pair of openchannel fermionic atoms $c_{k \sigma}$ and a closed-channel diatomic molecule $b_{q}$. The form of this atom-molecule coupling guarantees that the Cooper-pair and molecular superfluids must condense together, and that, in $d>1$, tuning through an s-wave FR leads to a smooth BECBCS crossover (rather than a phase transition) between two limits of a single paired superfluid state.

However, in 1d long-range order (BEC) is precluded even at $T=0$ by enhanced quantum fluctuations [16], and superfluidity is characterized by a quasi-long-ranged superfluid order, that admits a richer set of possibilities. To see this, it is convenient to use a bosonized representation [17, 18] of the atomic and molecular fields, with

$$
\begin{aligned}
b(x) & \sim \mathrm{e}^{i \phi_{b}(x)}, \\
c_{\sigma}(x) & \sim \mathrm{e}^{i k_{f} x} \mathrm{e}^{i\left(\phi_{\sigma}(x)+\theta_{\sigma}(x)\right)}+\mathrm{e}^{-i k_{f} x} \mathrm{e}^{i\left(\phi_{\sigma}(x)-\theta_{\sigma}(x)\right)},
\end{aligned}
$$

an approximate form sufficient here. In the above $k_{f}$ is the Fermi wavevector, the two terms in Eq. (3) are the right and left moving contributions to $c_{\sigma}$, and $\theta_{\sigma}$ and $\phi_{\sigma}$ are the fermion charge-density wave (phonon) and superfluid phase fields, respectively. These can be conveniently split into charge $(c)$ and spin $(s)$ components via $\phi_{c, s}=\left(\phi_{\uparrow} \pm \phi_{\downarrow}\right) / \sqrt{2}$ and similarly for $\theta_{c, s}$.

Using Eqs.(23) inside $H$ and focussing on densities incommensurate with the optical lattice, we find the coherent-state imaginary-time action (with $\hbar=1$ )

$$
\begin{aligned}
S & =\int d x d \tau\left(\sum_{\alpha=b, c} \frac{K_{\alpha}}{2 \pi}\left[v_{\alpha}\left(\partial_{x} \phi_{\alpha}\right)^{2}+v_{\alpha}^{-1}\left(\partial_{\tau} \phi_{\alpha}\right)^{2}\right]\right. \\
& +\frac{1}{2 \pi K_{s}}\left[v_{s}\left(\partial_{x} \theta_{s}\right)^{2}+v_{s}^{-1}\left(\partial_{\tau} \theta_{s}\right)^{2}\right]+\frac{g_{b s}}{2 \pi a^{2}} \cos \sqrt{8} \theta_{s} \\
& \left.-\frac{g_{\mathrm{fr}}}{\pi a^{2}} \cos \left(\phi_{b}-\sqrt{2} \phi_{c}\right) \cos \sqrt{2} \theta_{s}\right),
\end{aligned}
$$

governing the dynamics of three acoustic modes $\left(\phi_{b}, \phi_{c}\right.$, and $\theta_{s}$ ) coupled by two key nonlinearities $g_{b s}$ and $g_{\mathrm{fr}}$, that arise from the backscattering part of fermionic shortrange interaction $\tilde{g}_{b s} n_{\uparrow}(x) n_{\downarrow}(x)$ and from the FR coupling, respectively. Here, the dimensionless couplings $g_{b s}=\tilde{g}_{b s} / \pi v_{f}$ and $g_{\mathrm{fr}}=2 \tilde{g}_{\mathrm{fr}} \sqrt{\rho_{b}} / \pi v_{f} \rho_{f}$, with $\rho_{f}$ and $\rho_{b}$ the detuning-dependent fermion and boson densities, $v_{f}=\pi \rho_{f} / 2 m$ the Fermi velocity. All other relevant atomic and molecular interactions have been incorporated into the Luttinger parameters $K_{b}, K_{c}$ and
$K_{s}$, and velocities $v_{b}, v_{c}$ and $v_{s}$. For weak interactions $K_{s, c} \approx 1 \pm g_{b s} / 2$ and $K_{b} \rightarrow \infty$. In the above $a=\left(\pi v_{f}^{1 / 2} \rho_{f}\right)^{-1}$ is the ultra-violet (UV) cutoff.

Based on experience with the sine-Gordon model 12 , 18] we expect and find that over a large range of Luttinger parameters $K_{\alpha}$ a pertubative treatment of the backscattering and FR nonlinearities breaks down. A renormalization group (RG) treatment is therefore necessary to ascertain the low-energy behavior of the system. This amounts to successively integrating out the high-energy degrees of freedom in an infinitesimal wavevector shell $a^{-1} e^{-\ell}<q<a^{-1}$ around the UV cutoff. Resulting effective couplings appearing in $S$ then determine the lowenergy thermodynamics of the system.

Before discussing the full behavior it is convenient to consider a limiting regime of the above model. The simplest case is that of large attractive atomic interactions, $g_{b s} \ll-1$ (that, as we will see below, is induced by a finite FR scattering even if the nonresonant atomic interaction $g_{b s}$ is moderately repulsive). In this limit, the backscattering nonlinearity "freezes" $\theta_{s}$ at 0 , corresponding to a spin-gap state with gap $\Delta_{b s} \approx 2 \sqrt{\left|g_{b s}\right| v_{s} K_{s}} / a$. Using $\theta_{s}=0$ inside $S$ reduces the problem to two acoustic modes $\phi_{b, c}$ coupled by a single FR nonlinearity. Its effect can be assessed using an RG analysis that here amounts to a determination of the scaling dimension $D$ of the FR operator $\mathcal{O}(x) \equiv \cos \left(\phi_{b}(x)-\sqrt{2} \phi_{c}(x)\right) \cos \sqrt{2} \theta_{s}(x)$ around the Gaussian fixed point, $g_{b s}=0, g_{\text {fr }}=0$. This in turn is determined by the long-scale behavior of $\left\langle\mathcal{O}(x) \mathcal{O}^{\dagger}(0)\right\rangle \sim|x|^{-2 D}$. A simple calculation in the spin-gap phase gives $D=D_{\mathrm{fr}} \equiv\left(4 K_{b}\right)^{-1}+\left(2 K_{c}\right)^{-1}$. A standard RG analysis 12 then shows that for $D_{\text {fr }}<2$ $\left(D_{\mathrm{fr}}>2\right)$ the effective $\mathrm{FR}$ coupling grows (diminishes) at low energies under the RG coarse-graining procedure. For $D_{\mathrm{fr}}<2$ and weak $g_{\mathrm{fr}} \ll 1$, on scales longer than $\xi_{\mathrm{fr}} \approx a v_{f}^{1 / 2} g_{\mathrm{fr}}^{-\frac{1}{2-D_{\mathrm{fr}}}}$, the FR coupling dominates over the kinetic energy and the growth of $g_{\mathrm{fr}}$ saturates at $g_{\mathrm{fr}}^{\frac{2}{2-D_{\mathrm{fr}}}}$. In this strong-coupling regime, the FR interaction locks the closed-channel molecular superfluid phase $\phi_{b}$ to the open-channel Cooper-pair superfluid phase $\sqrt{2} \phi_{c}$, a characteristic of the "coupled spin-gap" (CSG) state. Approximating the FR coupling by a harmonic spring $\left(g_{\mathrm{fr}}^{\frac{2}{2-D_{\mathrm{fr}}}} / 2 \pi a^{2}\right)\left(\phi_{b}-\sqrt{2} \phi_{c}\right)^{2}$, with a "stiffness" softened by quantum fluctuations up to the scale $\xi_{\text {fr }}$, and diagonalizing the quadratic form in $\phi_{b}$ and $\phi_{c}$, gives two dispersions of the CSG state:

$$
\begin{aligned}
& \omega_{ \pm}(k)^{2}=\frac{1}{2}\left[\left(v_{c}^{2}+v_{b}^{2}\right) k^{2}+\Delta_{\mathrm{fr}}^{2}\right. \\
& \left.\quad \mp \sqrt{\left[\left(v_{c}^{2}+v_{b}^{2}\right) k^{2}+\Delta_{\mathrm{fr}}^{2}\right]^{2}-4 v_{c}^{2} v_{b}^{2} k^{4}-4 c^{2} k^{2} \Delta_{\mathrm{fr}}^{2}}\right],
\end{aligned}
$$

with $\Delta_{\mathrm{fr}} \equiv a^{-1}\left|g_{\mathrm{fr}}\right|^{\frac{1}{2-D_{\mathrm{fr}}}} \sqrt{v_{b} K_{b}^{-1}+2 v_{c} K_{c}^{-1}}$, and $c \equiv$ $\sqrt{\left(v_{c} K_{c}+2 v_{b} K_{b}\right) /\left(v_{c}^{-1} K_{c}+2 v_{b}^{-1} K_{b}\right)}$. The dispersion $\omega_{+}(k)(\approx c k)$ characterizes the gapless Bogoliubov mode 
corresponding to in-phase oscillations of the closedchannel molecular $\left(\phi_{b}\right)$ and the open-channel Cooper-pair $\left(\phi_{c}\right)$ superfluid phases. The dispersion $\omega_{-}\left(\approx \Delta_{\mathrm{fr}}\right)$ is for the gapped mode (in the long wavelength limit given by $\left.\phi_{-} \equiv \phi_{b}-\sqrt{2} \phi_{c}\right)$ in which $\phi_{b}$ and $\phi_{c}$ fluctuate out phase. Hence, the CSG state is characterized by two gapped modes $\theta_{s}, \phi_{-}$and a single in-phase gapless Bogoliubov mode (see Fig. 1b). In this coupled state the molecular phase $\phi_{b}$ is characterized by an effective Luttinger parameter $\bar{K}_{b}=\sqrt{\left(v_{b} K_{b}+v_{c} K_{c} / 2\right)\left(v_{b}^{-1} K_{b}+v_{c}^{-1} K_{c} / 2\right)}>K_{b}$ that can be read off from the action, Eq. (44) after simply imposing the low-energy FR coupling constraint $\phi_{c}=\phi_{b} / \sqrt{2}$ inside $S$. Similarly, atomic charge correlations are controlled by $\bar{K}_{c}=2 \bar{K}_{b}$.

Now consider $D_{\text {fr }}>2$ but still deep within the spingapped state, $g_{b s} \ll-1$. Here, quantum fluctuations of $\phi_{b, c}$ become sufficiently strong so as to average away longscale effects of the FR coupling, reducing it relative to the kinetic energy of fluctuations on these long scales. We refer to this distinct thermodynamic state (special to 1d) as the "decoupled spin-gap" (DSG) superfluid. It is characterized by effectively independent fluctuations of the low energy molecular and Cooper-pair superfluid phases and therefore exhibits two gapless superfluid modes displayed in Fig. 1t (with velocities $v_{b}$ and $v_{c}$ ), observable via Bragg spectroscopy [10].

Hence through the decoupling transition charge Luttinger parameters "jump" from $\bar{K}_{b, c}$ down to $K_{b, c}$. This is reflected in the corresponding momentum distribution functions $n_{b, c}(k)$, that for molecules [using Eq. (2)] is given by $n_{b}(k) \propto \int d x \mathrm{e}^{i k x}\left\langle b^{\dagger}(x) b(0)\right\rangle \sim k^{-\alpha}($ for $k \rightarrow 0)$, with $\alpha_{\text {coupled }}=1-\left(2 \bar{K}_{b}\right)^{-1}>\alpha_{\text {decoupled }}=1-\left(2 K_{b}\right)^{-1}$. This abrupt enhancement in the low $k$ peak of $n_{b}(k)$ reflects the suppression of molecular phase fluctuations in the coupled phase (due to locking to $\phi_{c}$ ) and should be measurable via time-of-flight images $[8]$.

Hence, as advertised in Fig.1, we predict a quantum phase transition between the coupled, CSG and decoupled, DSG spin-gapped superfluids. To determine the phase diagram outside of the deep spin-gap regime, i.e., for values of the backscattering amplitude $g_{b s}$ other than $g_{b s} \ll-1$, requires a detailed RG analysis. As outlined above the RG computation involves progressively integrating out (perturbatively in $g_{\mathrm{fr}}$ and $g_{b s}$ ) degrees of freedom at the UV cutoff scale $a^{-1}$. For simplicity we specialize here to the case of equal velocities $\left(v_{b} \simeq v_{c} \simeq v_{s}\right)$, leaving the more technically challenging general case to a future study [15]. The result of this coarse-graining procedure is summarized by the RG flow equations

$$
\begin{aligned}
& \frac{d g_{b s}}{d \ell}=2 g_{b s}\left(1-K_{s}\right)-\frac{1}{2} g_{\mathrm{fr}}^{2}, \\
& \frac{d g_{\mathrm{fr}}}{d \ell}=\left(2-D_{\mathrm{fr}}-\frac{1}{2} K_{s}\right) g_{\mathrm{fr}}-\frac{1}{2} g_{\mathrm{fr}} g_{b s},
\end{aligned}
$$

$$
\begin{aligned}
\frac{d K_{s}}{d \ell} & =-\frac{1}{2} g_{b s}^{2}-\frac{1}{4} g_{\mathrm{fr}}^{2}, \\
\frac{d D_{\mathrm{fr}}}{d \ell} & =-\frac{9}{8} g_{\mathrm{fr}}^{2} .
\end{aligned}
$$

One important subtlety [18] is that abelian bosonization does not explicitly capture the underlying $\mathrm{SU}(2)$ spinrotation symmetry embodied in our system's Hamiltonian. It can, however, be restored (as we have done above) by a specific choice of the UV cutoff $a$ and by imposing the relation $K_{s}=1+g_{b s} / 2$ on the initial conditions for the flows. This constraint stems from the fact that the value of $g_{b s}$ and the correction to $K_{s}$ arise from the same fermion interaction. It is simple to verify that this $\mathrm{SU}(2)$ invariance relation between $K_{s}$ and $g_{b s}$ is preserved by the RG flows Eq. (6) and (8), i.e., that for $K_{s}=1+g_{b s} / 2$ initially, $d\left(1+g_{b s} / 2-K_{s}\right) / d \ell=$ $g_{b s}\left(1+g_{b s} / 2-K_{s}\right)=0$.

Incorporating this relation significantly simplifies the RG flow equations, giving:

$$
\begin{aligned}
\frac{d g_{b s}}{d \ell} & =-g_{b s}^{2}-g_{\mathrm{fr}}^{2} / 2, \\
\frac{d g_{\mathrm{fr}}}{d \ell} & =\left(3 / 2-D_{\mathrm{fr}}-3 g_{b s} / 4\right) g_{\mathrm{fr}},
\end{aligned}
$$

along with Eq. (9) which is unchanged. The phase diagram Fig. 1is determined by the asymptotic (large $\ell$, corresponding to low energies and long scales) flows of $g_{\mathrm{fr}}(\ell)$, $D_{\text {fr }}(\ell)$ and $g_{b s}(\ell)$. A priori, one might have expected four distinct phases corresponding to four different combinations of relevent and irrelevant regimes of two couplings $g_{b s}$ and $g_{\mathrm{fr}}$. However, as is clear from the structure of the flow equations [particularly Eq. (10)], it is impossible to have a phase-coupled but spin-gapless state characterized by an asymptotically nonzero $g_{\mathrm{fr}}(\ell \rightarrow \infty)$ and a vanishing $g_{b s}$. That is, back-scattering $g_{b s}$ (that leads to Cooper-pair singlet formation) is always relevant when $g_{\mathrm{fr}}$ is. Physically this can be understood by observing directly from $S$ that an arbitrary strength FR coupling, at $T=0$ always induces pairing in an itinerant (i.e., ignoring Mott-insulating effects of a commensurate lattice) fermionic atom system.

One simple limit of the flow equations is $D_{\mathrm{fr}} \gg 1$, in which case $g_{\text {fr }}$ clearly flows to 0 and the phase boundary separating the DSG (for $g_{b s}<0$ ) and decoupled spingapless DNG (for $g_{b s}>0$ ) states asymptotes to $g_{b s}=$ 0 , respectively corresponding to attractive and repulsive nonresonant atomic interactions.

Another observation is that, for large repulsive background interactions $g_{b s} \gg 1$, the phase boundary separating the CSG and DNG states asymptotes to $D_{\mathrm{fr}}=$ $3 / 2$, as can be seen from the flow equations. These show that a strong background repulsion strongly suppresses the FR coupling $g_{\text {fr }}$ through Eq. (11), so that it has little effect on the flow of $g_{b s}$, which then itself can flow to 0 along the $g_{\mathrm{fr}} \approx 0$ sine-Gordon separatrix. This then reduces the eigenvalue for $g_{\text {fr }}$ in Eq. (11) to $3 / 2-D_{\text {fr }}$ leading 
to the phase boundary $D_{\mathrm{fr}}=3 / 2$. This is consistent with the observation that the FR coupling scaling dimension $D=D_{\mathrm{fr}}+\frac{1}{2} K_{s}$ is 2 at the transition and $K_{s}=1$ in the DNG state, constrained by the spin-rotational invariance. For an intermediate and small positive values of $g_{b s}$, we have numerically integrated the $\mathrm{RG}$ equations to determine the interpolation of the vertical part $\left(D_{\mathrm{fr}}=3 / 2\right)$ to the horizontal part $\left(g_{b s}=0\right)$ of the phase boundary, as illustrated in Fig. [1]

In contrast to repulsive background interactions that can be irrelevant if the FR coupling is, attractive interaction $\left(g_{b s}<0\right)$ always grows under coarse-graining. Physically, this is a reflection that Cooper-pairing always takes place at $T=0$, even in the absence of the FR coupling that can only enhance spin-singlet formation. Hence, at sufficiently long scales, the RG flows leave their perturbative regime of validity confined to $\left|g_{b s}\right| \lesssim 1$ and $\left|g_{\text {fr }}\right| \lesssim 1$. Outside of this range requires a nonperturbative analysis. As discussed earlier, the deep spin-gap state $g_{b s} \ll-1$ can nevertheless be analyzed by simply setting $\theta_{s}=0$ in the action and recomputing the flows perturbatively in $g_{\mathrm{fr}}$. As illustrated in Fig. 1 this leads to a phase boundary between CSG and DSG that is asymptotically vertical and given by $D_{\text {fr }}=2$.

To calculate this phase boundary for more moderate (but still negative) values of $g_{b s}$ requires a "matching" calculation. To see this we note that for small negative $g_{b s}$, perturbative RG flows (describing spin fluctuations on shorter length scales inside the spin-gapped state), remain valid up to a crossover spin-gap length scale, $\xi_{b s} \approx a v_{s}^{1 / 2}\left|g_{b s}\right|^{-1 /\left(2-2 K_{s}\right)}$ (coinciding with the width of the soliton in the sine-Gordon model). Hence we integrate the perturbative $\mathrm{RG}$ flows out to length $\xi_{b s}=a v_{s}^{1 / 2} e^{\ell_{*}}$, defined by $g_{b s}\left(\ell^{*}\right) \approx 1$, beyond which spin fluctuations freeze out, $\theta_{s} \approx 0$. On longer scales we match onto the strongly coupled spin-gapped state, characterized by setting $\theta_{s}=0$ inside the action, Eq. (44) but with the fluctuation-renormalized parameters $g_{\mathrm{fr}}\left(\ell^{*}\right)$ and $D_{\mathrm{fr}}\left(\ell^{*}\right)$ determined by the RG flow. Setting $D_{\mathrm{fr}}\left(\ell^{*}\right)=2$ then determines the fluctuation-renormalized CSG-DSG phase boundary illustrated in Fig. 1 The shift of the phase boundary toward a larger critical $D_{\text {fr }}$ can be seen from the decrease of $D_{\mathrm{fr}}(\ell)$ under coarse-graining, which in turn corresponds to enhancement of superfluidity by the FR coupling.

A nontrivial challenge is the experimental realization of a LL with parameters tunable across the decoupling transition. The necessary large value of $D_{\mathrm{fr}}>3 / 2$, requires small Luttinger parameters, $K_{\alpha}$, realized by strongly interacting systems, for which a relation to microscopic parameters is difficult to establish. One exception is the extended quarter-filled 1d Hubbard model, where in the limit of large on-site repulsion, $K_{c}=\left[2+4 / \pi \sin ^{-1} v\right]^{-1}$, with $v=V /(2|t|)$ a ratio (limited to $<1$ by phase separation) of the nearest neighbor hopping $t$ and repulsion $V$ energies, respectively [19]. Hence, $K_{c}$ as low as a $1 / 4$ can be reached, assuring a transition (that for strong repulsion takes place at $D_{\mathrm{fr}}=3 / 2$ ) into the decoupled superfluid state at $v=1 / \sqrt{2}$ for weakly interacting molecules. In the more favorable molecular Tonks regime with $K_{b} \rightarrow 1$, we predict the quantum transition at $v \approx 0.38$. Thus we suggest that a Feshbach-resonantly interacing atomic gas confined in a highly anisotropic (1d) trap and subjected to a periodic optical potential [8] is a promising candidate for an experimental realization of the phase diagram and the decoupling transition discussed here.

We gratefully acknowledge discussions with Victor Gurarie and Matthew Fisher, as well as support from NSF DMR-0321848 and the Packard Foundation.

[1] C.A. Regal et al., Phys. Rev. Lett. 92, 040403 (2004); M.W. Zwierlein et al., Phys. Rev. Lett. 92, 120403 (2004); J. Kinast et al., Phys. Rev. Lett. 92, 150402 (2004); M. Bartenstein et al., Phys. Rev. Lett. 92, 203201 (2004).

[2] E. Timmermans et al., Phys. Lett. A 285, 228 (2001); M. Holland et al., Phys. Rev. Lett. 87, 120406 (2001); Y. Ohashi and A. Griffin, Phys. Rev. Lett. 89, 130402 (2002); J. Stajic, et al., Phys. Rev. A 69, 063610 (2004).

[3] This is a reasonable assumption even in $1 \mathrm{~d}$ in most currently realized experimental systems [1] characterized by a strong FR coupling (i.e., with width much larger than the Fermi energy), so that closed and open channels are strongly hybridized precluding an unabiguous separation between molecules and Cooper pairs. The latter is, however, possible for narrow FR[4].

[4] K. E. Strecker et al., Phys. Rev. Lett. 91, 080406 (2003).

[5] A. Recati et al., Phys. Rev. Lett. 90, 020401 (2003).

[6] A. Recati et al., Phys. Rev. A 71, 033630 (2005).

[7] M.A. Cazalilla, J. Phys. B 37, S1 (2004).

[8] B. Paredes et al., Nature (London) 429, 277 (2004).

[9] H. Moritz et al, cond-mat/0503202

[10] J. Steinhauer et al., Phys. Rev. Lett. 90, 060404 (2003); S. Richard et al., Phys. Rev. Lett. 91, 010405 (2003).

[11] See, e.g., D. Jaksch et al., Phys. Rev. Lett. 81, 3108 (1998); L.-M. Duan et al., Phys. Rev. Lett. 91, 090402 (2003).

[12] J. V. José et al., Phys. Rev. B 16, 1217 (1977).

[13] C.S. O'Hern et al., Phys. Rev. Lett. 83, 2745 (1999).

[14] V.J. Emery et al., Phys. Rev. Lett. 85, 2160 (2000).

[15] D.E. Sheehy and L. Radzihovsky, in preparation.

[16] P.C. Hohenberg, Phys. Rev. 158, 383 (1967).

[17] F.D.M. Haldane, Phys. Rev. Lett. 47, 1840 (1981).

[18] See, e.g., T. Giamarchi, Quantum Physics in One Dimension (Clarendon, Oxford, 2004).

[19] H.J. Schulz, Phys. Rev. Lett. 64, 2831 (1990). 